\title{
Patterns of Inpatient Antibiotic Use Among Public Hospitals in Hong Kong from 2000 to 2015
}

\author{
Celine S. L. Chui $i^{1,2,3,4,5} \cdot$ Benjamin J. Cowling ${ }^{1}$ (1) $\cdot$ Wey Wen Lim ${ }^{1} \cdot$ Christopher K. M. Hui ${ }^{6} \cdot$ Esther W. Chan $^{2}$. \\ lan C. K. Wong ${ }^{2,5,7} \cdot$ Peng Wu ${ }^{1}$
}

Published online: 12 March 2020

(c) Springer Nature Switzerland AG 2020

\begin{abstract}
Background Studies have demonstrated that higher rates of antibiotic resistance are found in countries with higher antibiotic consumption. The global increase in antibiotic consumption is a major public health concern.

Objectives The objective of this study was to describe the antibiotic dispensing patterns in public hospitals in Hong Kong from 2000 to 2015.

Methods We acquired data on all hospital admissions with any antibiotics dispensed from 2000 to 2015 from the Hong Kong public hospitals. The annual proportion of hospital admissions with antibiotics dispensed was estimated and stratified by age group. An interrupted time series analysis was conducted to examine any potential change in tetracycline dispensing after the release of the new clinical practice guideline.

Results A total of 35,535,506 antibiotic prescriptions were dispensed among 2,161,360 unique hospitalized patients from 2000 to 2015 . Antibiotics were dispensed in $29.2 \%$ of all hospital admissions in the public hospitals, the annual proportions of hospital admissions with antibiotics dispensed increased over the study period from $27.87 \%$ in 2000 to $31.39 \%$ in 2015 , ranging from 27.17 to $31.39 \%$. However, a significant increase was only observed in age groups of 5-19, 20-44, and 85 years or above when stratifying by age. In the interrupted time series analysis, a change in trend was detected for tetracycline dispensing that coincided with the time of publication of the new clinical practice guideline.

Conclusions We found that the overall volume of antibiotic use increased between 2000 and 2015. The rise in the dispensing of carbapenems in our study is concerning. The significant change in tetracycline use after being recommended as one of the preferred regimens demonstrated that the change in the clinical practice guideline had an immediate effect on the antibiotic prescribing practice in Hong Kong public hospitals.
\end{abstract}

\section{Key Points}

The overall volume of antibiotics consumed in Hong Kong increased in the 16 years between 2000 and 2015.

At least one antibiotic was dispensed in $29.2 \%$ of all hospital admissions.

Local clinical practice guidelines appeared to influence the actual prescribing practice.

Benjamin J. Cowling

bcowling@hku.hk

Extended author information available on the last page of the article

\section{Introduction}

Antimicrobial resistance (AMR) is a pressing global health concern $[1,2]$. Some common infections such as sinusitis, pneumonia, and acute otitis media are becoming more difficult to treat because of AMR [3]. In 2017, the World Health Organization (WHO) published a list of drug-resistant bacteria ranked according to the potential threat posed to human health [4]. The list included bacterial families that are resistant to the last-resort antibiotic classes such as carbapenems. Overuse of antibiotics is thought to be one of the major drivers of AMR [5]. Another study also showed that carriage of resistant bacteria is associated with the usage of antibiotics [6]. In addition, higher rates of antibiotic resistance are found in countries with higher antibiotic consumption [7]. Therefore, the use of antibiotics and AMR is closely related.

In the past decade, antibiotic consumption has increased by $65 \%$ globally, with a particularly concerning increase 
in the use of last-resort antibiotic drug classes including carbapenems and polymyxins [8, 9]. In 2013, the WHO organized the first meeting to work on the strategic plan and priority activities to tackle AMR; the subsequent endorsement of the Global Action Plan on Antimicrobial Resistance was proposed in May 2015 [1]. Since then, there have been sparse longitudinal data on antibiotic use. There is an urgent need to examine antibiotic prescribing patterns after the adoption of this global action plan. According to the statistics from the Department of Health of Hong Kong, the number of community-associated methicillin-resistant Staphylococcus aureus (MRSA) cases notified to the Centre for Health Protection of the Department of Health has increased five-fold from 2008 to 2016. When compared to other countries, the proportions of resistant bacterial isolates were at a high level in Hong Kong in both Gram-positive and Gram-negative bacteria, as indicated in the data on $S$. aureus resistant to methicillin and Escherichia coli resistant to third-generation cephalosporins and fluoroquinolones from the Hong Kong Hospital Authority [10]. In light of the emergence of resistant pathogens, the Centre for Health Protection, Health Authority, and experts from local universities published the Inter-hospital Multi-disciplinary Programme on Antimicrobial ChemoTherapy (IMPACT) guideline [11], which is a collection of evidence-based recommendations for clinical antibiotic prescribing in combating AMR in the clinical setting. The IMPACT guideline was first released in 1999; the fifth edition published in 2017 being the latest. Throughout the years, new editions of IMPACT were published with changes in recommendation on prescribing practices based on new evidence. The effect of such changes on the actual prescribing practice should also be examined. This study aims to describe the antibiotic dispensing patterns in public hospitals in Hong Kong from 2000 to 2015 and the characteristics of antibiotic users.

\section{Methods}

\subsection{Sources of Data}

We acquired data on all hospital admissions with any antibiotics dispensed from 2000 to 2015 from the Hospital Authority in Hong Kong. The Hospital Authority is a statutory body that manages the public healthcare sector in Hong Kong. Its services are available to all Hong Kong citizens (approximately 7.5 million population) at minimal out-ofpocket cost. More than $80 \%$ of hospital admissions in Hong Kong are captured in the public sector [12]. Therefore, the source population of this dataset included individuals who had access to public healthcare services in Hong Kong. All prescriptions are dispensed through a centralized management system in the public hospitals managed by the Hospital
Authority. The data used in this study were retrieved from this management system.

All dispensed antibiotics were recorded with details such as unique patient identification number, hospital admission year and month, antibiotic prescription year and month, prescription order (if multiple antibiotics were prescribed), prescribed antibiotic name, frequency, and duration in days. Information was also available on the primary discharge diagnosis and up to an additional 14 diagnoses of each hospital admission. All diagnoses were coded according to the International Classification of Diseases, Ninth Revision, Clinical Modification (ICD-9-CM) and were available up to three digits. Medication classes were recorded following the British National Formulary Classification [13]. Similar data collected from the Hospital Authority were previously used in epidemiological studies and found to have a high positive predicated value in various diagnoses [14, 15], which demonstrated the validity of the data for conducting the current study.

We also acquired the aggregated weekly hospital admission data stratified by age from the hospital authority. In the case where a week spanned over 2 months, the number of hospital admissions in each month was counted proportionately based on the number of days in each corresponding month. Age groups were defined as $0-4,5-19,20-44$, $45-64,65-84$, and 85 years or above.

\subsection{Data Analysis}

\subsubsection{Patterns in Antibiotic Use}

Antibiotic use was defined as having at least one dispensing record of antibiotics within a hospital admission. We divided the number of admissions with antibiotics dispensed by the overall number of hospital admissions in each year to calculate the annual proportion of hospital admissions with antibiotics dispensed. We also calculated the annual proportion stratified by age group. The switch of route of administration was also examined.

We examined the number of drug classes dispensed in each hospital admission by categorizing antibiotics into the following classes: narrow-spectrum penicillins, broadspectrum penicillins, $\beta$-lactamase inhibitor combinations, first- and second-generation cephalosporins, third- and fourth-generation cephalosporins, fifth-generation cephalosporins, carbapenems, tetracyclines, aminoglycosides, macrolides, lincosamides, polymixins, sulfonamides, metronidazole and tinidazole, quinolones, and other antibiotics. The classification used in this study was based on the previous literature $[8,16]$ on the prescribing trends in other countries for an easy comparison and the special interest in the resistance against last-resort antibiotics [17] such as carbapenems and polymixins. We calculated the proportion of 
hospital admissions with different numbers of drug classes prescribed among all admissions with any antibiotic dispensed. The frequency of first prescriptions of the aforementioned antibiotics by drug class within each hospital admission was also examined because frequent use of first-line antibiotics may indicate a good adherence to the prescription guidelines.

The proportion of dispensed antibiotics listed in the reserve group of the WHO Model List of Essential Medicines [18] was also estimated by dividing the annual number of hospital admissions with reserve antibiotics dispensed by the total number of hospital admissions of the corresponding year. The list of antibiotics that were in the reserve group and available in the Hospital Authority within the study period includes: aztreonam, cefepime, ceftaroline, daptomycin, fosfomycin, linezolid, polymyxins (including colistin and polymyxin B), and tigecycline.

The IMPACT fourth edition was published in November 2012 [19], which included the suggestion to replace macrolides with tetracycline as one of the preferred regimens for hospitalized community-acquired pneumonia owing to the emergence of macrolide-resistant Mycoplasma pneumoniae, while the preferred regimens was co-amoxiclav with a macrolide in the third edition of IMPACT in 2005. An interrupted time series analysis was therefore conducted with the data from 2000 through 2015 to examine the potential change in tetracycline and macrolide dispensing among public hospitals before and after the release of the fourth edition of the guideline. The monthly proportion of hospital admissions with tetracycline dispensed was used to measure the tetracycline use in the inpatients. A segmented linear regression was conducted using the R-package "segmented". The breakpoint was automatically estimated in the model rather than being pre-specified. If a significant change in the dispensing trend was identified shortly after the publication of the updated IMPACT guideline (fourth edition), it might indicate that changes in the guideline did have an impact on the prescribing practices in the public hospitals.

\subsubsection{Diagnoses Associated with Antibiotic Use}

We measured the duration of antibiotic use by estimating the days of therapy (DOT) per hospital bed-day [20] for admissions related to bacterial infection based on the primary discharge diagnosis coded by ICD-9-CM. Primary diagnosis was considered to ensure the antibiotic prescription was dispensed for treatment of the primary infections rather than for treatment of secondary infections or prophylaxis. These diagnoses were categorized into the following infection groups based on the previous literature [21]: (1) Pneumonia (ICD-9: 481-486); (2) skin and subcutaneous infections (ICD-9: 680-686); and (3) septicemia (ICD-9: 038). Although the WHO recommends the defined daily dose as the unit for drug utilization studies [22], it only reflects drug use for defined major indications among adults. Therefore, the DOT was used in this study instead to examine the drug utilization among patients of different age groups. To estimate the annual DOT per hospital bed-day, we summed the prescription duration in days of all antibiotic prescriptions for admissions with the above-selected bacterial infections as the primary discharge diagnosis and divided by the sum of hospital bed-days of these admissions. To ensure the validity and reliability of the data on prescription duration, only antibiotics dispensed on or after year 2004 were included in this part of the analysis. Dispensing records with a missing prescription duration in days were excluded from this analysis. All analyses were conducted using R 3.1.2 (R Foundation for Statistical Computing, Vienna, Austria) and SAS 9.4 (SAS Inc., Cary, NC, USA).

\section{Results}

A total of $35,535,506$ antibiotic prescriptions were dispensed among 2,161,360 unique hospitalized patients in 6,091,580 hospital admissions from 2000 to 2015. Antibiotics were dispensed in $29.2 \%$ of all hospital admissions in the public hospitals, the annual proportions of hospital admissions with antibiotics dispensed increased over the study period from $27.87 \%$ in 2000 to $31.39 \%$ in 2015 , ranging from 27.17 to $31.39 \%$. The number of antibiotics dispensed among the inpatients during admissions into Hong Kong public hospitals from 2000 through 2015 are listed in Appendix 1. The most commonly dispensed antibiotics were amoxicillin-clavulanate (co-amoxiclav), cefuroxime, metronidazole, piperacillin-tazobactam, levofloxacin, cloxacillin, ampicillin, cefoperazone, ceftriaxone, and ciprofloxacin. These ten antibiotics accounted for $68 \%$ of all antibiotics dispensed to the inpatients admitted during the study period. A total of $15 \%$ of admissions had antibiotic prescriptions with a switched route of administration from oral to parenteral, whilst around 29\% switched from parenteral to oral within one admission. When comparing the first and last antibiotic prescriptions within the same hospital admission, 20.3\% switched from parenteral to oral whilst $57.3 \%$ remained the same route (38.7\% for oral and $18.6 \%$ for parenteral). The most common primary discharge diagnoses were listed in Table 1 for all hospital admissions with antibiotics dispensed. Pneumonia was the most common diagnosis with an antibiotic dispensed, followed by chronic airway obstruction. General symptoms, the fifth most common diagnosis associated with antibiotic dispensing, did not provide adequate details of the hospital admission. Therefore, we examined the second-listed diagnosis among hospitalizations with general symptoms as the primary discharge diagnosis (Appen$\operatorname{dix} 2$ ). 
Table 1 Top ten primary discharge diagnoses among hospital admissions with antibiotics dispensed

\begin{tabular}{lll}
\hline ICD-9-CM diagnostic codes & Frequency $(n)$ & Proportion (\%) \\
\hline 486 Pneumonia organism unspecified & 502,214 & 8.27 \\
496 Chronic airway obstruction not elsewhere classified & 328,080 & 5.40 \\
599 Other disorders of urethra and urinary tract & 305,609 & 5.03 \\
682 Other cellulitis and abscess & 146,498 & 2.41 \\
780 General symptoms & 135,847 & 2.24 \\
038 Septicemia & 111,683 & 1.84 \\
428 Heart failure & 108,239 & 1.78 \\
519 Other diseases of respiratory system & 107,063 & 1.76 \\
585 Chronic kidney disease (CKD) & 92,677 & 1.53 \\
162 Malignant neoplasm of trachea bronchus and lung & 88,686 & 1.46
\end{tabular}

ICD-9-CM International Classification of Diseases, Ninth Revision, Clinical Modification
Overall, at least one antibiotic was dispensed in $29.2 \%$ of the hospital admissions (in total 20,853,366 hospital admissions from 2000 to 2015), and the annual proportions of hospital admissions with antibiotics dispensed increased over the study period. When stratified by age, statistically significant increased trends were observed among the age groups of $5-19,20-44$, and 85 years or above $(p<0.05)$ (Fig. 1). Among all age groups, patients aged 85 years or above were most likely to have antibiotic treatment when hospitalized, with antibiotic treatment dispensed in more than half of such admissions (Fig. 1).
Among all the hospital admissions with antibiotic(s) dispensed, more than $50 \%$ had only one antibiotic drug class dispensed throughout the admission (Appendix 3), which was equivalent to $18 \%$ of all the hospital admissions. Among the hospital admissions with one antibiotic drug class only, $58 \%$ were dispensed with B-lactamase inhibitors combinations. The most commonly dispensed antibiotics as the first prescription were from the drug class of ß-lactamase inhibitor combinations (Fig. 2). Over the study period, apart from broad-spectrum penicillins (including ampicillins and amoxicillin), all the drug classes were increasingly dispensed as the first antibiotic in the hospital admissions. Meanwhile, a

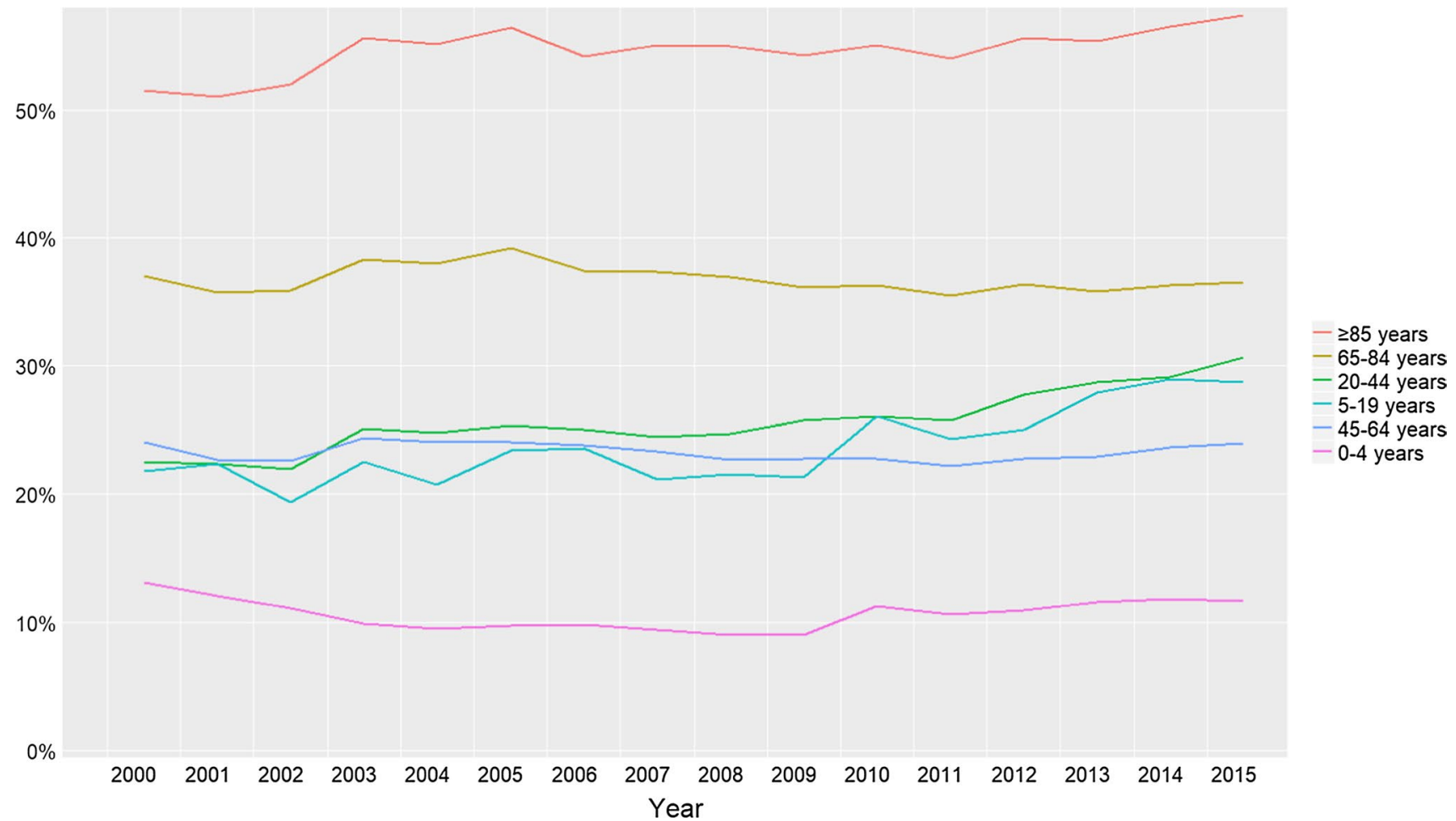

Fig. 1 Proportion of hospital admissions with antibiotics dispensed from 2000 to 2015 by age 


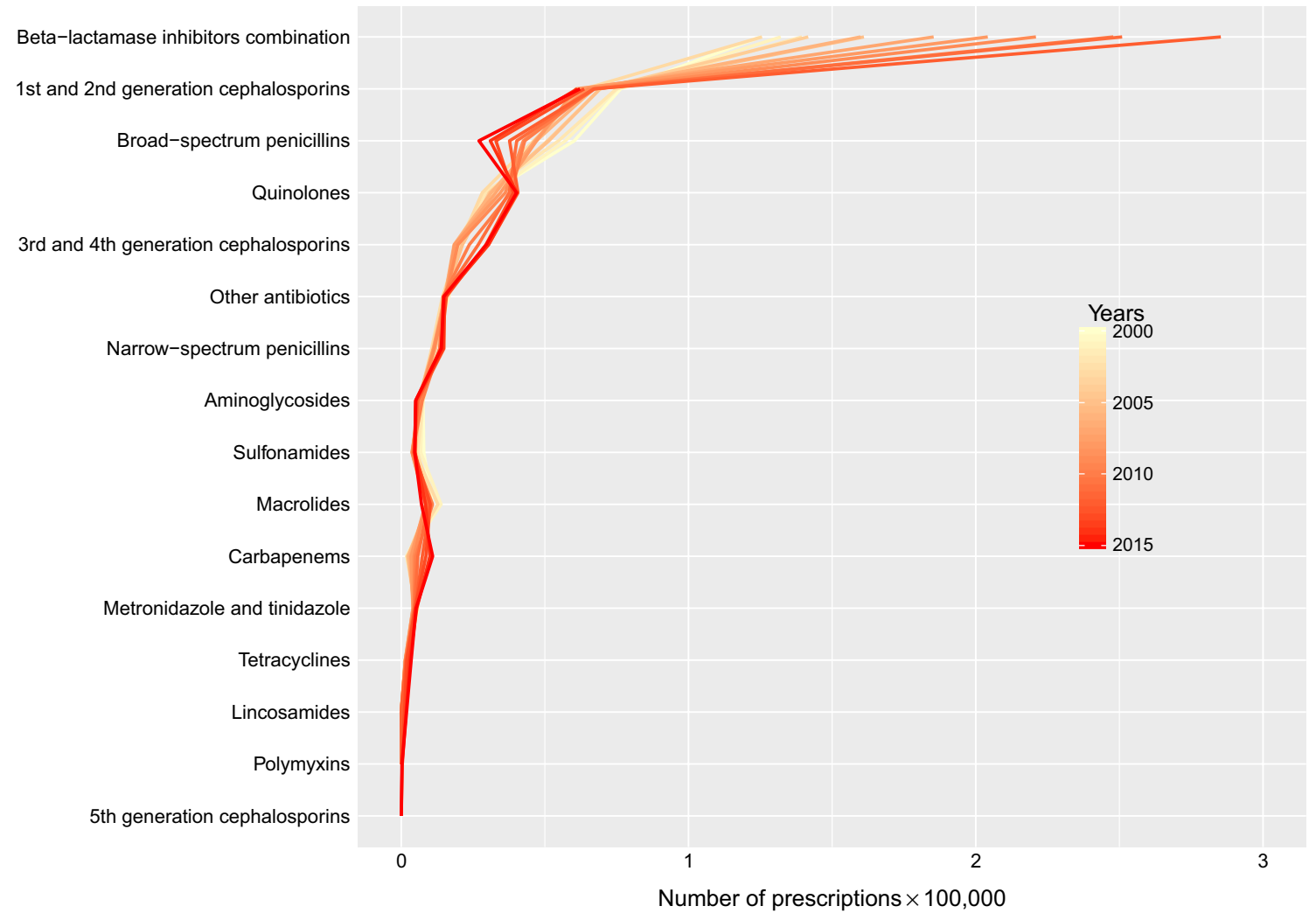

Fig. 2 Frequency of drug classes of the first antibiotic dispensed in each hospital admission from 2000 to 2015

rapid elevation in the dispensing volume was observed in B-lactamase inhibitor combinations.

Over the 16-year period, a total of 49,735 hospital admissions $(0.24 \%$ of overall hospital admissions; $0.82 \%$ of all hospital admissions with antibiotic dispensed) had at least one record of a dispensed antibiotic in the reserve group. The frequency of annual prescriptions of reserve antibiotics had been increasing over time in general, except for cefepime, which however was the most frequently dispensed reserve antibiotic (Fig. 3). The most common primary discharge diagnosis associated with prescription of cefepime was pneumonia (16.46\%). Only one dispensing record of polymyxin B was identified, which was included in the polymyxins group together with colistin in Fig. 3 .

In the interrupted time series analysis on the monthly proportions of hospital admissions (per 1000 admissions) with tetracycline dispensed (Fig. 4a), the change in trend was detected in November 2012 (turning point), which coincided with the publication month of the IMPACT fourth edition. The slopes of the fitted linear trends before and after the turning point were -0.003 and 0.127 , respectively, which were significantly different from each other $(p=0.044)$. A similar analysis was conducted for macrolides where no similar change in the trend was observed at the same time period (Fig. 4b).
From 2004 to 2015, the total hospital admissions with the three pre-defined bacterial infections as the primary discharge diagnosis accounted for $15.1 \%$ of all admissions having antibiotics dispensed. Admissions with diagnoses of pneumonia and septicemia have twice the DOT as compared with admissions with a diagnosis of skin and subcutaneous infections. Meanwhile, the antibiotic utilization patterns were similar between these two types of admissions (Fig. 5). Admissions with the primary diagnosis of skin and subcutaneous infections had the shortest average number of bed-days per admission among the three bacterial infections (Table 2). Co-amoxiclav was the most commonly dispensed antibiotic for admissions associated with pneumonia and septicemia while the inpatients with skin and soft-tissue infections most often received cloxacillin for treatment (Table 2).

\section{Discussion}

We described the overall antibiotic use in Hong Kong public hospitals with more than 35 million records of antibiotic dispensing from 2000 to 2015 . We found that the overall volume of antibiotic use had increased over the 16 years. When stratified by age, there were no major increases in 
Fig. 3 Proportion of hospital admissions with dispensing of antibiotics in the World Health Organization (WHO) reserve group from 2000 to 2015

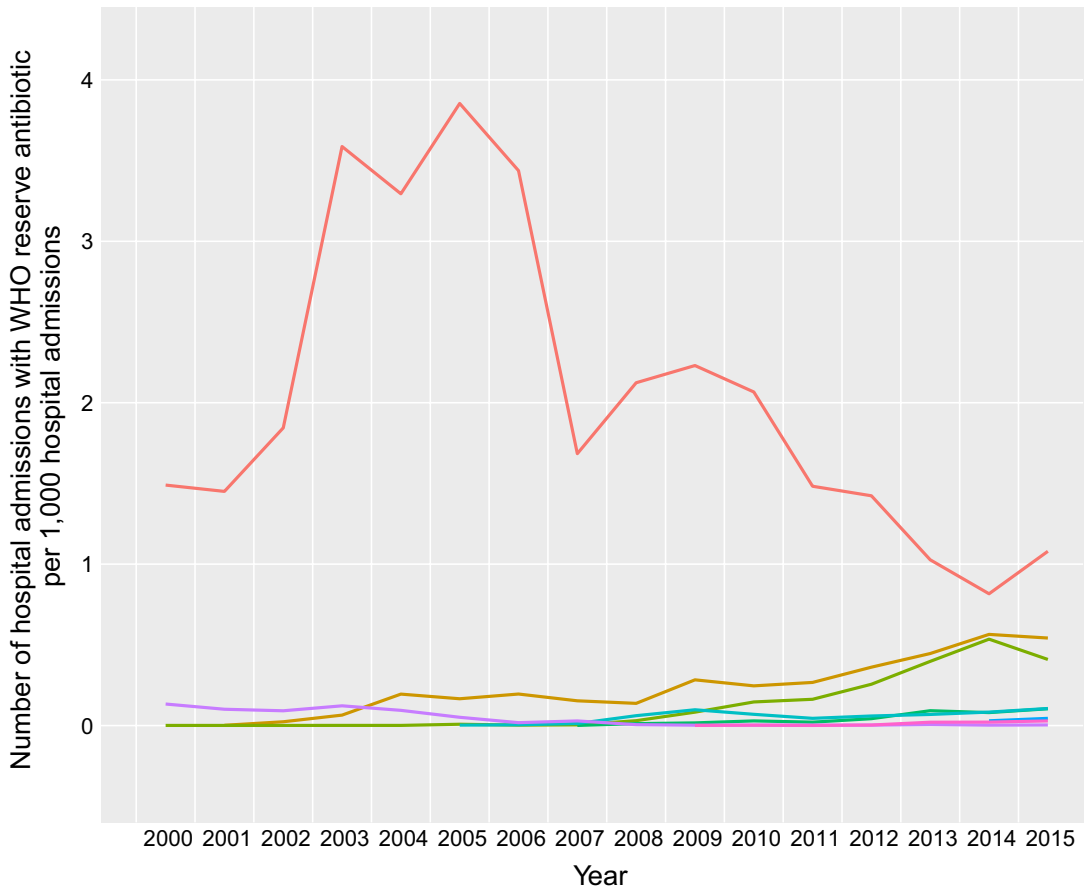

Reserve antibiotics - Cefepime - Linezolid - Polymyxins - Daptomycin - Tigecycline - Cefetaroline - Aztreonam - Fosfomycin the proportions of admissions with antibiotics dispensed in all age groups. The increase in the dispensed B-lactamase inhibitor combinations may account for the overall increase in antibiotic use in all admissions given that the majority of the prescriptions were co-amoxiclav. The average overall proportion of hospital admissions with at least one antibiotic prescription (29.2\%) estimated in our study was slightly lower than that (34.4\%) from the recently published Global Point Prevalence Survey study based on the data collected from 53 countries in 2015 [23], while penicillins in combination with $\beta$-lactamase inhibitors were the most commonly dispensed antibiotic drug class in both studies.

The primary discharge diagnoses associated with hospitalization with antibiotics dispensed were mostly infections such as pneumonia, other disorders of the urethra and urinary tract (inclusive of urinary tract infection), and cellulitis. Chronic airway obstruction was the second most common diagnosis among all hospitalizations with antibiotics dispensed. As infection is a known common co-morbidity of chronic airway obstruction, the antibiotics that were dispensed in these episodes might be for the treatment of such a co-morbidity $[24,25]$. The most common secondary diagnoses of hospitalization with "general symptoms" as the primary discharge diagnosis included essential hypertension, diabetes mellitus, and some other non-specific conditions (Appendix 2).

We observed an increasing proportion of admissions with only one drug class of antibiotics dispensed over the study period. This might indicate a decreasing treatment failure rate, or antibiotics being used for prophylaxis. Factors contributing to treatment failure include bacterial resistance, appropriateness of empirical therapy, and patients' adherence to antibiotic therapy [3]. However, we would not be able to determine the occurrence of antibiotic treatment failure and the underlying factors associated with the treatment failure (if any) while the observed reduction in the number of drug classes dispensed over time might indicate an improvement in management of hospitalized patients.

Previous studies suggested that the use of antibiotics was one of the factors associated with carriage of antibioticresistant bacteria [26-28], and overuse of antibiotics might drive the observed increase in AMR [5]. In addition, bacterial load in MRSA carriers was indicated to be related to the use of fluoroquinolones and B-lactam therapy [29]. Therefore, the rise in dispensing of $\beta$-lactams including carbapenems in our study was concerning especially when carbapenems, as one of the last-resort antibiotics, had been increasingly dispensed as the first antibiotic dispensed over the study period. The decrease in the use of broad-spectrum penicillins as first-line antibiotics was probably owing to the preference for B-lactamase inhibitor combinations, such as co-amoxiclav, with a broader spectrum against Gramnegative bacteria. This might also indicate an increasing prevalence of AMR in the hospital setting or a conservative prescribing behavior in choosing broad-spectrum antibiotics. A previous study showed that inappropriate empirical antibiotic treatment was an independent risk factor for 30-day mortality in patients with Klebsiella pneumoniae bacteremia [30]. Given the rapidly increasing use of $\beta$-lactamase inhibitor combination drugs such as co-amoxiclav as a first-line 
Fig. 4 Proportion of hospital admissions with a tetracycline and $\mathbf{b}$ macrolides dispensed from 2000 to 2015 . Note the spike in 2003 associated with a large local epidemic of severe acute respiratory syndrome

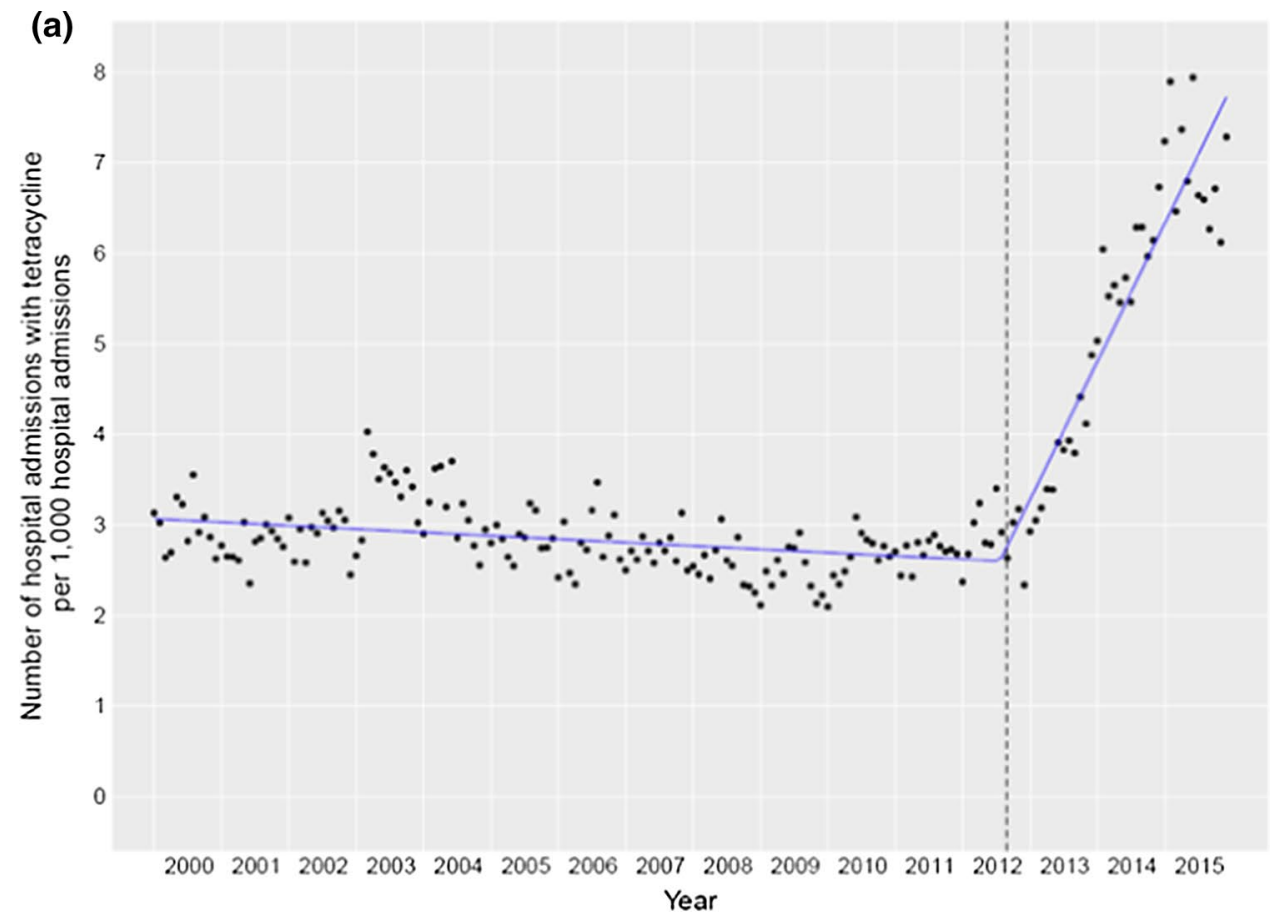

(b)

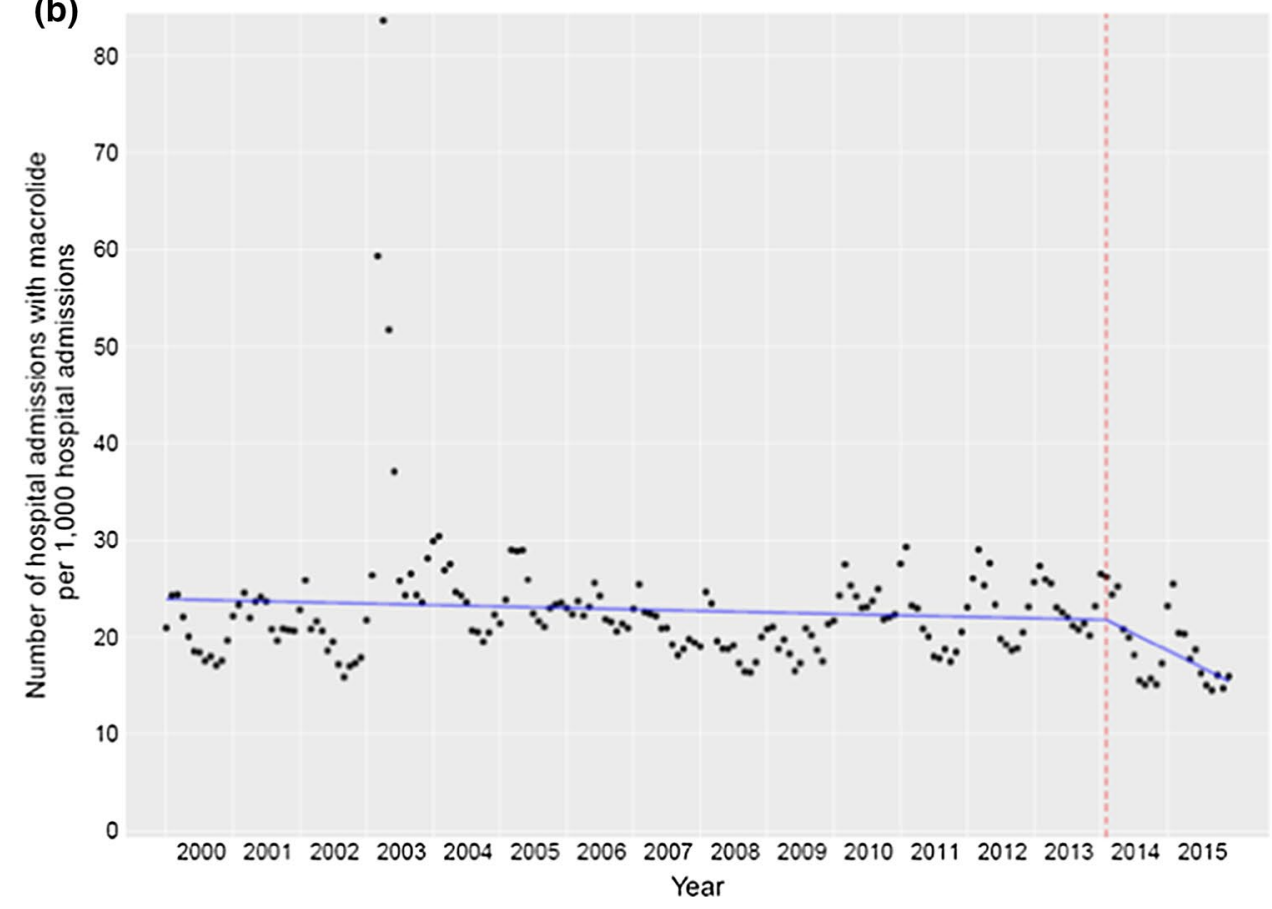

drug, further investigation into the appropriate use of antibiotics may be needed to prevent the development of resistance and improve the outcome of clinical treatment. It is also worth noting that in addition to clinical use of antibiotics in humans, multiple factors might have been driving the development of AMR including antibiotics used in agriculture and veterinary medicine $[31,32]$. Stakeholders should work together within the one-health framework to effectively combat AMR.
Our study showed that the use of the WHO reserve antibiotics in Hong Kong generally increased from 2000 to 2015 except for cefepime with a decline in prescription after 2006, coinciding with the publication of the consensus statement of the antimicrobial stewardship program in Hong Kong [33]. Linezolid, indicated to treat MRSA infections, was the second most commonly dispensed WHO reserve antibiotic in this study. The increasing dispensing of linezolid was largely consistent with the change in the prevalence 
Fig. 5 Days of therapy per bed-day for admissions with the primary discharge diagnosis of pneumonia, skin and subcutaneous infections, and septicemia

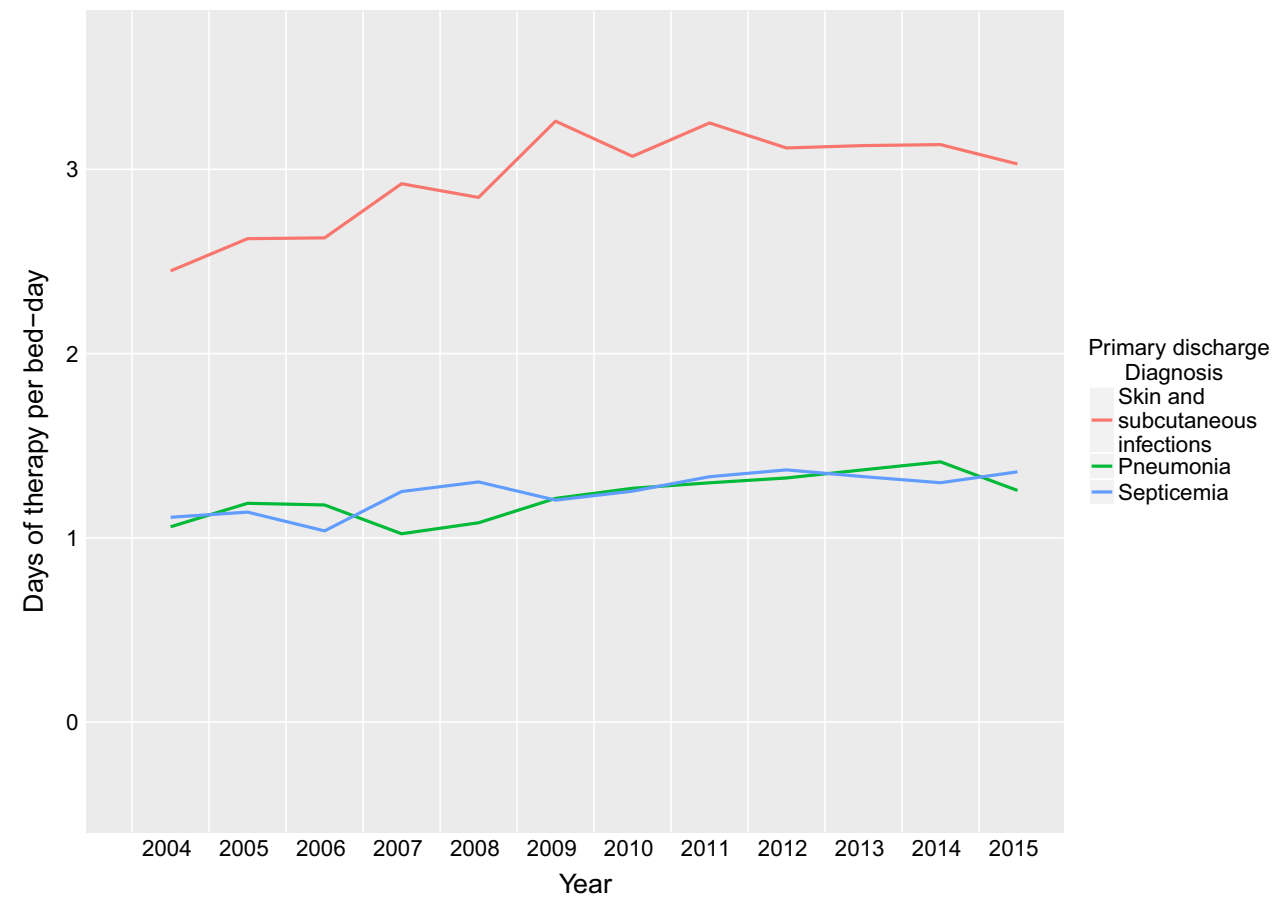

Table 2 Characteristics of hospital admissions with pneumonia, skin and subcutaneous infections, and septicemia as the primary discharge diagnosis

\begin{tabular}{|c|c|c|c|c|c|c|}
\hline & & & Pneumonia & \multicolumn{2}{|c|}{$\begin{array}{l}\text { Skin and subcutaneous infec- } \\
\text { tions }\end{array}$} & Septicemia \\
\hline \multicolumn{3}{|l|}{ No. of hospital admissions } & 505,563 & \multicolumn{2}{|l|}{134,839} & 91,752 \\
\hline \multicolumn{3}{|l|}{ No. of patients } & 285,221 & \multicolumn{2}{|l|}{102,048} & 72,788 \\
\hline \multicolumn{3}{|l|}{ Male $(\%)$} & 54.14 & \multicolumn{2}{|l|}{56.97} & 48.55 \\
\hline \multicolumn{3}{|c|}{ Average no. of antibiotic prescriptions per hospital admission } & 6.84 & \multicolumn{2}{|l|}{7.11} & 7.76 \\
\hline \multicolumn{3}{|c|}{ Average no. of drug classes per hospital admission } & 1.67 & \multicolumn{2}{|l|}{1.92} & 1.76 \\
\hline \multicolumn{3}{|c|}{ Average no. of hospital bed-days per hospital admission } & 11.25 & \multicolumn{2}{|l|}{6.09} & 10.80 \\
\hline \multicolumn{7}{|c|}{ Top 5 most commonly dispensed antibiotics } \\
\hline Generic names & Frequency $^{\mathrm{a}}$ & $\%$ & Frequency $^{\mathrm{a}}$ & $\%$ & Frequency $^{\mathrm{a}}$ & $\%$ \\
\hline Ampicillin & 13,087 & 0.38 & 259,515 & 22.48 & 28,381 & 3.99 \\
\hline Cefoperazone & 182,258 & 5.27 & 6597 & 0.69 & 39,035 & 4.56 \\
\hline Cefuroxime & 62,147 & 1.80 & 15,464 & 1.61 & 76,653 & 8.95 \\
\hline Clarithromycin & 210,435 & 6.08 & 3811 & 0.40 & 6796 & 0.95 \\
\hline Co-amoxiclav & $1,399,208$ & 40.45 & 229,387 & 19.87 & 240,991 & 28.15 \\
\hline Cloxacillin & 27,535 & 0.80 & 325,394 & 28.18 & 17,281 & 2.43 \\
\hline Gentamicin & 42,688 & 1.23 & 8808 & 0.92 & 46,411 & 5.42 \\
\hline Levofloxacin & 170,656 & 4.93 & 32,649 & 2.83 & 27,767 & 3.90 \\
\hline Metronidazole & 66,598 & 1.93 & 35,214 & 3.05 & 26,385 & 3.70 \\
\hline Piperacillin + tazobactam & 317,336 & 9.17 & 12,625 & 1.32 & 45264 & 5.29 \\
\hline
\end{tabular}

${ }^{\mathrm{a}}$ Each admission normally had multiple prescriptions for one antibiotic

of MRSA in Hong Kong from 2.8 to $21.6 \%$ between 2005 and 2011 [34, 35]. Although the observed associations [6, $7,36]$ might not necessarily indicate a causal relationship between antibiotic exposure and development of resistance, the use of the WHO reserve antibiotics should be continuously monitored. 
With this large dataset, we were able to identify an effect of the change in clinical practice guideline on the actual dispensing patterns in hospitals. In the interrupted time series analysis, tetracycline use increased significantly after being added as one of the preferred regimens of the fourth IMPACT guideline published in 2012, whilst during the same time no change was observed in macrolide prescriptions, which presumably should not be affected by the guideline update. Tetracycline remained the preferred regimens for hospitalized community-acquired pneumonia in the latest edition of the IMPACT guideline [11]. Our analysis demonstrated that the change in the clinical practice guideline had an immediate effect on the antibiotic prescribing practice. Should there be any need to change the prescribing practice in the future, changing the clinical practice guideline in public hospitals can be considered as one of the channels for better implementation.

The estimated DOT indicated that on average there was more than one antibiotic prescription per hospital-day for admissions due to pneumonia, skin and subcutaneous infections, and septicemia, which appeared to be in line with the recommendation of having combined antibiotic treatments $[11,19]$. Admissions related to skin and subcutaneous infections had the highest DOT because of the shortest hospitalday but the highest number of antibiotics dispensed among these admissions. In line with the empirical treatment recommended by the local IMPACT guideline [11], co-amoxiclav and cloxacillin were the most commonly dispensed antibiotics for pneumonia and skin and soft-tissue infections, respectively, indicating a good adherence to local clinical practice guidelines among public hospitals in Hong Kong.

There were several limitations of this study. First, the primary discharge diagnostic codes might not be the indication for the dispensed antibiotics. Based on the currently available data, we were unable to determine the indication for which the antibiotic was dispensed. Therefore, we could not rule out the possibilities that some antibiotics were dispensed for infection control or surgical prophylaxis. This is a common limitation of using healthcare records to conduct population-based studies. However, we are confident that the study population had good compliance with the antibiotics dispensed based on stringent medication administration protocols within the hospital. Therefore, the dispensing records should closely reflect the actual usage among the inpatients. Second, this study only included antibiotic use data from public hospitals in Hong Kong. According to the recently published wholesale supply data of antibiotics in Hong Kong [37], the Hospital Authority is the second largest sector, purchasing $20 \%$ of all antibiotics supplied in Hong Kong, whilst only $4-5 \%$ were supplied to private hospitals. This ratio of antibiotic supply to public versus private hospitals is consistent with the fact that around $80 \%$ of all local hospital admissions occur in public hospitals and $20 \%$ occur in private hospitals. However, more than $50 \%$ of the antibiotics supplied to the outpatient sector were consumed by the private general practitioners. Further studies on antibiotic use in the private outpatient healthcare sector are urgently needed.

\section{Conclusions}

To the best of our knowledge, this is the first study that examined the dispensing records of antibiotics in Hong Kong. A general increasing trend of antibiotic usage was indicated in patients admitted to public hospitals. Significant temporal changes were observed in the prescription of some drug classes. The recent increase in the use of lastresort antibiotic class such as carbapenems is concerning. This study suggested that local clinical practice guidelines appeared to influence the actual prescribing practice in hospitals. Future policies in changing clinical practice with antibiotics may be implemented through modifying the recommendations in the clinical practice guidelines.

Acknowledgements The authors thank Dr. Pak-Leung Ho for clarifying the exact publication month and release of IMPACT fourth edition; and Ms. Julie Au for technical assistance.

\section{Compliance with Ethical Standards}

Funding This work was financially supported by a commissioned grant from the Health and Medical Research Fund (Grant No. HKS-16-E10).

Conflict of Interest Benjamin J. Cowling reports receipt of honoraria from Roche and Sanofi Pasteur. Celine S.L. Chui, Wey Wen Lim, Christopher K.M. Hui, Esther W. Chan, Ian C.K. Wong, and Peng Wu have no conflicts of interest that are directly relevant to the content of this article.

\section{Appendix 1}

See Table 3. 
Table 3 Characteristics of antibiotics dispensed in hospital admissions from 2000 to 2015

\begin{tabular}{|c|c|c|c|c|}
\hline Characteristics & $\begin{array}{l}\text { Number of anti- } \\
\text { biotic prescrip- } \\
\text { tions }\end{array}$ & $\begin{array}{l}\text { Number of } \\
\text { admissions }\end{array}$ & Number of patients & $\begin{array}{l}\text { Proportion of hospital admis- } \\
\text { sions with antibiotic prescrip- } \\
\text { tions (\%) }\end{array}$ \\
\hline \multicolumn{5}{|l|}{ Years } \\
\hline 2000 & $1,628,578$ & 317,008 & 203,720 & 27.87 \\
\hline 2001 & $1,775,288$ & 325,408 & 162,598 & 27.17 \\
\hline 2002 & $1,789,236$ & 323,501 & 142,714 & 27.14 \\
\hline 2003 & $1,756,413$ & 291,123 & 118,844 & 29.42 \\
\hline 2004 & $1,886,998$ & 318,387 & 121,977 & 29.10 \\
\hline 2005 & $1,942,394$ & 334,138 & 121,160 & 29.99 \\
\hline 2006 & $1,895,268$ & 325,024 & 114,764 & 29.13 \\
\hline 2007 & $1,998,396$ & 345,052 & 115,264 & 29.04 \\
\hline 2008 & $2,070,466$ & 362,897 & 116,484 & 28.82 \\
\hline 2009 & $2,217,885$ & 383,579 & 121,601 & 28.67 \\
\hline 2010 & $2,451,864$ & 418,448 & 130,534 & 29.39 \\
\hline 2011 & $2,438,512$ & 421,294 & 129,300 & 28.56 \\
\hline 2012 & $2,676,392$ & 459,141 & 140,390 & 29.59 \\
\hline 2013 & $2,798,301$ & 462,451 & 137,012 & 30.00 \\
\hline 2014 & $3,017,105$ & 489,230 & 141,080 & 30.69 \\
\hline 2015 & $3,192,410$ & 515,128 & 143,918 & 31.39 \\
\hline \multicolumn{5}{|l|}{ Sex } \\
\hline Male & $19,776,291$ & $3,230,425$ & $1,081,522$ & \\
\hline Female & $15,759,215$ & $2,861,384$ & $1,079,838$ & \\
\hline \multicolumn{5}{|c|}{ Age group (years) } \\
\hline $0-4$ & $1,803,260$ & 276,972 & 195,141 & \\
\hline $5-19$ & $1,075,676$ & 872,941 & 141,783 & \\
\hline $20-44$ & $4,349,029$ & $1,243,018$ & 553,438 & \\
\hline $45-64$ & $7,806,668$ & 232,148 & 526,224 & \\
\hline $65-84$ & $14,807,418$ & $2,452,140$ & 606,466 & \\
\hline $85+$ & $5,692,372$ & $1,014,361$ & 138,089 & \\
\hline Unknown & 1083 & 229 & 219 & \\
\hline
\end{tabular}

\section{Appendix 2}

See Table 4.

Table 4 Top ten secondary diagnoses of the hospital admissions with general symptoms (ICD-9: 780) as the primary discharge diagnosis

\begin{tabular}{lcc}
\hline ICD-9-CM diagnostic code & Frequency $(n)$ & Proportion (\%) \\
\hline 401 Essential hypertension & 15,145 & 7.20 \\
250 Diabetes mellitus & 12,177 & 5.79 \\
599 Other disorders of urethra and urinary tract & 8033 & 3.82 \\
438 Late effects of cerebrovascular disease & 7094 & 3.37 \\
465 Acute upper respiratory infections of multiple or unspecified sites & 6065 & 2.88 \\
486 Pneumonia organism unspecified & 5686 & 2.70 \\
427 Cardiac dysrhythmias & 5197 & 2.47 \\
276 Disorders of fluid electrolyte and acid-base balance & 4852 & 2.31 \\
294 Persistent mental disorders due to conditions classified elsewhere & 4485 & 2.13 \\
780 General symptoms & 4066 & 1.93 \\
\hline
\end{tabular}

ICD-9-CM International Classification of Diseases, Ninth Revision, Clinical Modification 


\section{Appendix 3}

See Fig. 6.

Fig. 6 Proportion of hospital admissions with a different number of antibiotic drug classes dispensed among all hospital admissions with antibiotics dispensed from 2000 to 2015

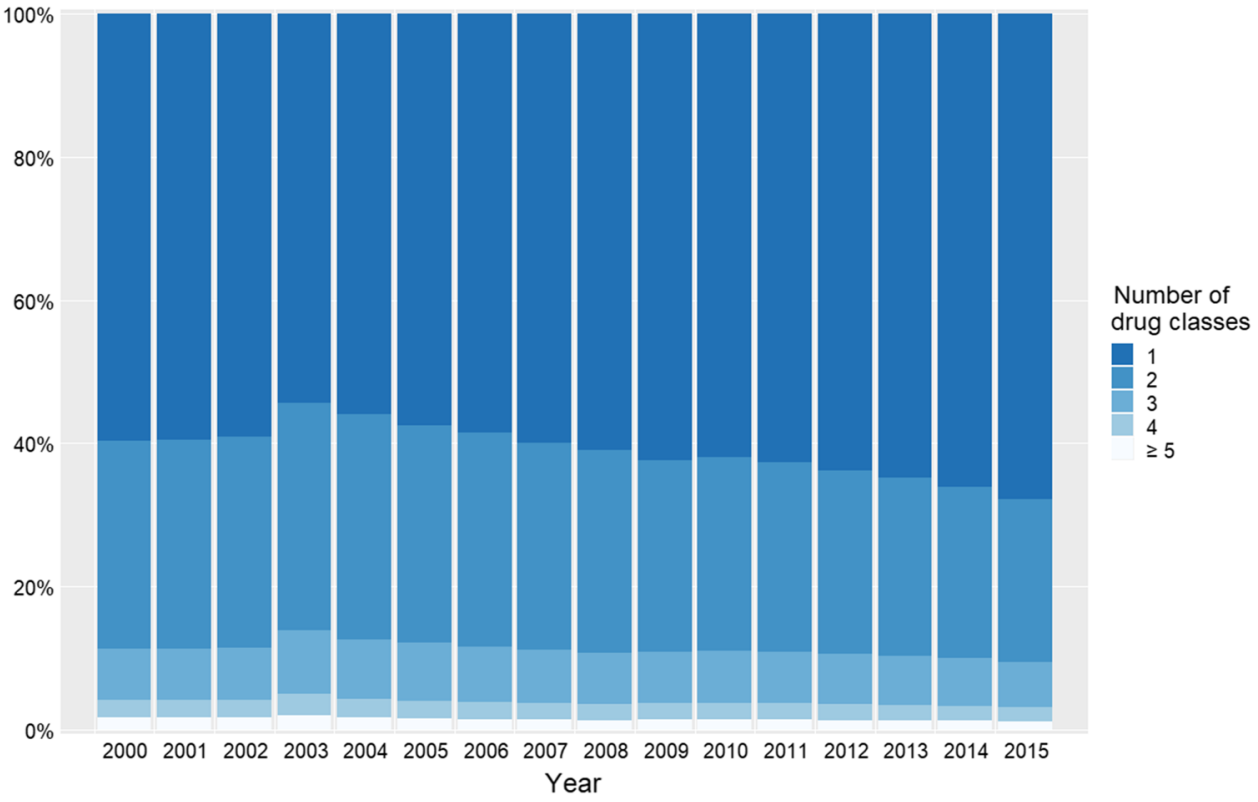

\section{References}

1. World Health Organization. Global action plan on antimicrobial resistance. Geneva, Switzerland, 2015. http://www.wpro.who.int/ entity/drug_resistance/resources/global_action_plan_eng.pdf. Accessed 28 Feb 2020.

2. O'Neill J. Antimicrobial resistance: tackling a crisis for the health and wealth of nations. 2014. https://amr-review.org/sites/default/ files/AMR\%20Review\%20Paper\%20-\%20Tackling\%20a\%20cri sis $\% 20$ for $\% 20$ the $\% 20$ health $\% 20$ and $\% 20$ wealth $\% 20$ of $\% 20$ nat ions_1.pdf. Accessed 28 Feb 2020.

3. Currie CJ, Berni E, Jenkins-Jones S, Poole CD, Ouwens M, Driessen S, et al. Antibiotic treatment failure in four common infections in UK primary care 1991-2012: longitudinal analysis. BMJ. 2014;349: g5493.

4. Willyard C. The drug-resistant bacteria that pose the greatest health threats. Nature. 2017;543(7643): 15.

5. Ventola CL. The antibiotic resistance crisis: part 1: causes and threats. P T. 2015;40(4):277-83.

6. van Bijnen EM, Paget J, de Lange-de Klerk ES, den Heijer CD, Versporten A, Stobberingh EE, et al. Antibiotic exposure and other risk factors for antimicrobial resistance in nasal commensal Staphylococcus aureus: an ecological study in 8 European countries. PLoS One. 2015;10(8):e0135094.

7. Goossens H, Ferech M, Stichele RV, Elseviers M, Grp EP. Outpatient antibiotic use in Europe and association with resistance: a cross-national database study. Lancet. 2005;365(9459):579-87.

8. Van Boeckel TP, Gandra S, Ashok A, Caudron Q, Grenfell BT, Levin SA, et al. Global antibiotic consumption 2000 to 2010: an analysis of national pharmaceutical sales data. Lancet Infect Dis. 2014;14(8):742-50.

9. Klein EY, Van Boeckel TP, Martinez EM, Pant S, Gandra S, Levin SA, et al. Global increase and geographic convergence in antibiotic consumption between 2000 and 2015. Proc Natl Acad Sci USA. 2018;115(15):E3463-70.

10. Centre for Health Protection. Hong Kong strategy and action plan on antimicrobial resistance 2017-2022. In: Department of Health, editor. Hong Kong. 2017.

11. Ho PL, Wu TC, Chao DVK, Hung IFN, Lui L, Lung DC, et al. Reducing bacterial resistance with IMPACT, 5th ed. Hong Kong. 2017, Centre for Health Protection, Department of Health.

12. Hong Kong Census and Statistics Department. Thematic household survey report no. 3. Hong Kong, 2000. https://www.statistics .gov.hk/pub/B11302032000XXXXB0100.pdf. Accessed 28 Feb 2020.

13. British Medical Association and Royal Pharmaceutical Society. British National Formulary United Kingdom: BMJ Group and Pharmaceutical Press. 2018. www.bnf.org. Accessed 28 Feb 2020.

14. Lau WC, Chan EW, Cheung C-L, Sing CW, Man KK, Lip GY, et al. Association between dabigatran vs warfarin and risk of osteoporotic fractures among patients with nonvalvular atrial fibrillation. JAMA. 2017;317(11):1151-8.

15. Wong AY, Root A, Douglas IJ, Chui CS, Chan EW, Ghebremichael-Weldeselassie Y, et al. Cardiovascular outcomes associated with use of clarithromycin: population based study. BMJ. 2016;352:h6926.

16. Baggs J, Fridkin SK, Pollack LA, Srinivasan A, Jernigan JA. Estimating national trends in inpatient antibiotic use among US hospitals from 2006 to 2012. JAMA Intern Med. 2016;176(11):1639-48.

17. Liu YY, Wang Y, Walsh TR, Yi LX, Zhang R, Spencer J, et al. Emergence of plasmid-mediated colistin resistance mechanism MCR-1 in animals and human beings in China: a microbiological and molecular biological study. Lancet Infect Dis. 2016;16(2):161-8.

18. World Health Organization. WHO model list of essential medicines. 2017. 20th list. http://www.who.int/medicines/publications/ 
essentialmedicines/20th_EML2017.pdf?ua=1. Accessed $28 \mathrm{Feb}$ 2020.

19. Ho PL, Wong SY, Hung IFN, Lung DC, Tsang KY, Wu TC, et al. Reducing bacterial resistance with IMPACT. 4th ed. 2012, Centre for Health Protection, Department of Health.

20. Polk RE, Fox C, Mahoney A, Letcavage J, MacDougall C. Measurement of adult antibacterial drug use in 130 US hospitals: comparison of defined daily dose and days of therapy. Clin Infect Dis. 2007;44(5):664-70.

21. Lee CC, Lee MT, Chen YS, Lee SH, Chen YS, Chen SC, et al. Risk of aortic dissection and aortic aneurysm in patients taking oral fluoroquinolone. JAMA Intern Med. 2015;175(11):1839-47.

22. World Health Organization. Introduction to drug utilization research. Oslo, Norway. 2003. http://apps.who.int/medicinedocs/ pdf/s4876e/s4876e.pdf. Accessed 28 Feb 2020.

23. Versporten A, Zarb P, Caniaux I, Gros MF, Drapier N, Miller M, et al. Antimicrobial consumption and resistance in adult hospital inpatients in 53 countries: results of an internet-based global point prevalence survey. Lancet Glob Health. 2018;6(6):e619-29.

24. Benfield T, Lange P, Vestbo J. COPD stage and risk of hospitalization for infectious disease. Chest. 2008;134(1):46-53.

25. Hopkinson NS, Molyneux A, Pink J, Harrisingh MC, Guideline C. Chronic obstructive pulmonary disease: diagnosis and management: summary of updated NICE guidance. BMJ. 2019;366:14486.

26. Cheng VCC, Chen JHK, So SYC, Wong SCY, Yan MK, Chau $\mathrm{PH}$, et al. Use of fluoroquinolones is the single most important risk factor for the high bacterial load in patients with nasal and gastrointestinal colonization by multidrug-resistant Acinetobacter baumannii. Eur J Clin Microbiol. 2015;34(12):2359-66.

27. Costelloe C, Metcalfe C, Lovering A, Mant D, Hay AD. Effect of antibiotic prescribing in primary care on antimicrobial resistance in individual patients: systematic review and meta-analysis. BMJ. 2010;340:c2096.

28. Furuno JP, McGregor JC, Harris AD, Johnson JA, Johnson $\mathrm{JK}$, Langenberg $\mathrm{P}$, et al. Identifying groups at high risk for carriage of antibiotic-resistant bacteria. Arch Intern Med. 2006;166(5):580-5.

29. Cheng VCC, Li IWS, Wu AKL, Tang BSF, Ng KHL, To KKW, et al. Effect of antibiotics on the bacterial load of meticillin-resistant Staphylococcus aureus colonisation in anterior nares. J Hosp Infect. 2008;70(1):27-34.

30. Man MY, Shum HP, Chan YH, Chan KC, Yan WW, Lee RA, et al. Clinical predictors and outcomes of Klebsiella pneumoniae bacteraemia in a regional hospital in Hong Kong. J Hosp Infect. 2017;97(1):35-41.

31. Levy SB, Marshall B. Antibacterial resistance worldwide: causes, challenges and responses. Nat Med. 2004;10(12 Suppl.):S122-9.

32. Holmes AH, Moore LS, Sundsfjord A, Steinbakk M, Regmi S, Karkey A, et al. Understanding the mechanisms and drivers of antimicrobial resistance. Lancet. 2016;387(10014):176-87.

33. Ho PL, Cheng JC, Ching PT, Kwan JK, Lim WW, Tong WC, et al. Optimising antimicrobial prescription in hospitals by introducing an antimicrobial stewardship programme in Hong Kong: consensus statement. Hong Kong Med J. 2006;12(2):141-8.

34. Ho PL, Wang TK, Ching P, Mak GC, Lai E, Yam WC, et al. Epidemiology and genetic diversity of methicillin-resistant Staphylococcus aureus strains in residential care homes for elderly persons in Hong Kong. Infect Control Hosp Epidemiol. 2007;28(6):671-8.

35. Cheng VC, Tai JW, Wong ZS, Chen JH, Pan KB, Hai Y, et al. Transmission of methicillin-resistant Staphylococcus aureus in the long term care facilities in Hong Kong. BMC Infect Dis. 2013;13:205.

36. Bell BG, Schellevis F, Stobberingh E, Goossens H, Pringle M. A systematic review and meta-analysis of the effects of antibiotic consumption on antibiotic resistance. BMC Infect Dis. 2014; $14: 13$.

37. Centre for Health Protection. Wholesale supply data of antibiotics in Hong Kong (2014-2016). Hong Kong, 2018. https://www.chp. gov.hk/en/static/100290.html. Accessed 28 Feb 2020.

\section{Affiliations}

\section{Celine S. L. Chui ${ }^{1,2,3,4,5} \cdot$ Benjamin J. Cowling ${ }^{1}$ - Wey Wen Lim ${ }^{1} \cdot$ Christopher K. M. Hui ${ }^{6}$ Esther W. Chan ${ }^{2}$. lan C. K. Wong ${ }^{2,5,7} \cdot$ Peng Wu ${ }^{1}$}

1 WHO Collaborating Centre for Infectious Disease Epidemiology and Control, School of Public Health, Li Ka Shing Faculty of Medicine, The University of Hong Kong, 1/F Patrick Manson Building (North Wing), 7 Sassoon Road, Hong Kong SAR, China

2 Department of Pharmacology and Pharmacy, Li Ka Shing Faculty of Medicine, Centre for Safe Medication Practice and Research, The University of Hong Kong, Hong Kong SAR, China

3 Department of Paediatrics and Adolescent Medicine, Li Ka Shing Faculty of Medicine, The University of Hong Kong, Hong Kong SAR, China
4 Department of Social Work and Social Administration, Faculty of Social Sciences, The University of Hong Kong, Hong Kong SAR, China

5 The University of Hong Kong-Shenzhen Hospital, Shenzhen, Guangdong, China

6 Department of Medicine, Li Ka Shing Faculty of Medicine, The University of Hong Kong, Hong Kong SAR, China

7 Research Department of Practice and Policy, UCL School of Pharmacy, London, UK 